\title{
Transmission ratio distortion in the human body louse, Pediculus humanus (Insecta: Phthiraptera)
}

\author{
CJ McMeniman and SC Barker \\ Parasitology Section, School of Molecular and Microbial Sciences, University of Queensland, Brisbane 4072, Australia
}

\begin{abstract}
We studied inheritance at three microsatellite loci in eight $F_{1}$ and two $F_{2}$ families of the body (clothes) louse of humans, Pediculus humanus. The alleles of heterozygous femaleparents were always inherited in a Mendelian fashion in these families. Alleles from heterozygous male-parents, however, were inherited in two different ways: (i) in a Mendelian fashion and (ii) in a non-Mendelian fashion, where males passed to their offspring only one of their two alleles,
\end{abstract}

that is, $100 \%$ nonrandom transmission. In male body lice, where there was non-Mendelian inheritance, the paternally inherited set of alleles was eliminated. We interpret this pattern of inheritance as evidence for extreme transmission ratio distortion of paternal alleles in this species.

Heredity (2006) 96, 63-68. doi:10.1038/sj.hdy.6800760; published online 21 September 2005

Keywords: lice; segregation distortion; meiotic drive; non-Mendelian inheritance

\section{Introduction}

Transmission ratio distortion (TRD) has been defined as statistically significant deviation from the ratio of offspring genotypes expected if inheritance was Mendelian (PardoManuel de Villena et al, 2000). In most instances of TRD, one member of a pair of heterozygous alleles or heteromorphic chromosomes is passed from either the male or female parent to offspring in excess of the expected Mendelian proportion of $50 \%$. Mechanistically, the unequal representation of parental alleles in offspring can result from (i) nonrandom segregation of chromosomes or alleles during meiosis (meiotic drive); (ii) postmeiotic selection which results in differential survival or fertilisation success of haploid gametes; or (iii) differential survival of diploid zygotes during development. Several cases of TRD of maternal and paternal alleles have been described in model organisms such as Drosophila spp, Mus spp, and Neurospora spp (see Lyttle, 1991 for a review). Recently, however, observations of novel types of TRD in other organisms have become more common, with the use of molecular markers in linkage analyses and population genetics studies (eg, Fishman et al, 2001).

Here, we report the discovery of TRD of paternal alleles in the body louse of humans, Pediculus humanus L. 1758, a diplodiploid insect that reproduces exclusively by sex. We determined the inheritance of alleles at three autosomal microsatellite loci in the $F_{1}$ and $F_{2}$ offspring of crosses between male and female body lice. The alleles of heterozygous female-parents at the three loci always were inherited in a Mendelian fashion. To our surprise, however, alleles of heterozygous male-parents were inherited in two ways in these families: (i) in a Mendelian

Correspondence: C McMeniman. Current address: School of Integrative Biology, University of Queensland, St Lucia, Brisbane Qld 4072, Australia. E-mail: c.mcmeniman@uq.edu.au

Received 4 June 2005; accepted 15 August 2005; published online 21 September 2005 fashion, and (ii) in a non-Mendelian fashion where one allele was not passed to the offspring ( $100 \%$ nonrandom transmission). Analysis of $\mathrm{F}_{2}$ offspring genotypes, in a cross where the parental origin of each of the male's two alleles was known, revealed that the set of alleles that was eliminated was of paternal origin (ie from the male's father). We interpret this pattern of inheritance as evidence for a novel and extreme case of TRD in male body lice. Hypotheses for the underlying mechanism of this unusual mode of inheritance in P. humanus are proposed.

\section{Materials and methods}

\section{Body lice}

Body lice were from the Orlando strain of $P$. humanus at the University of Queensland. This strain was founded with body lice from an unspecified number of people in Washington, DC and Orlando, FL, USA in March 1942 (Culpepper, 1944). The Orlando strain was kept at the United States Department of Agriculture, Bureau of Entomology and Plant Quarantine, Orlando, Florida from 1942 to 1945 by feeding the lice on human volunteers (Culpepper, 1944, 1946). The strain adapted to feeding on the blood of rabbits in 1945 (Culpepper, 1948) and has been fed on rabbit blood ever since. Lice were kept in an incubator at $30 \pm 1{ }^{\circ} \mathrm{C}, 50 \%$ relative humidity with a photoperiod of $3 \mathrm{~h}: 21 \mathrm{~h}$ (light:dark).

\section{Experimental crosses}

Pairs of virgin adult males and virgin adult females were put in $30 \mathrm{ml}$ glass bottles with a $3 \mathrm{~cm}^{2}$ of black cotton cloth, on which eggs were laid. Each pair of lice was fed on rabbit blood once each day. After 12-14 days of egg laying the male and female were removed and stored at $4^{\circ} \mathrm{C}$ in $100 \%$ ethanol. Eggs hatched in 7-9 days. The hatch rate of eggs from each cross was recorded. Nymphs moulted to adults in 12 days. These adults were then sexed and stored at $4^{\circ} \mathrm{C}$ in $100 \%$ ethanol. 
Families of lice

Eight $F_{1}$ families (families $1-8$ ) were bred. To determine the parental origin of the allele that was not passed from heterozygous male-parents to their offspring, heterozygous males from family 7, where the origin of each allele was known, were crossed with females from family 8 to produce two $F_{2}$ families (families 9 and 10). Lice were collected from the above $F_{1}$ families as third-stage nymphs to ensure virginity and then kept in separate bottles.

\section{Microsatellites}

Total genomic DNA was extracted from individual lice with a DNeasy Tissue Kit (Qiagen, Germany). Three microsatellite loci were amplified by PCR from each louse: ML8, ML9 and ML10 (Leo et al, 2005). Primer sequences and annealing temperatures $\left(T_{\mathrm{a}}\right)$ for each locus are as follows: $M L 8$ (for: AAA CGT TCG AAT GGG ACT TGA AC, rev: CCC ACA CAT AGC CGC CAT T, $T_{\mathrm{a}}=51{ }^{\circ} \mathrm{C}$ ); $M L 9$ (for: TCC ATC ATC CAT CGT TTC GA, rev: CCG ATT CTC CTT ATT CAT TCG C, $T_{\mathrm{a}}=51^{\circ} \mathrm{C}$ ); ML10 (for: GCC AAG GTC TAA TTA TCC, rev: GAA CGA GAA GAT GCG TAA ACG, $T_{\mathrm{a}}=42^{\circ} \mathrm{C}$ ). The reverse primer at each locus was labelled with HEX dye (Proligo, Germany) to allow fluorescent detection of PCR products. DNA was amplified in $12.5 \mu \mathrm{l}$ reactions containing $1.25 \mu \mathrm{l}$ of $10 \times$ Taq polymerase buffer $(\mathrm{AB}$ Gene, Epsom, UK), $2.5 \mathrm{mM}$ of $\mathrm{MgCl}_{2}, 0.22 \mathrm{mM}$ of dNTPs, $0.12 \mu \mathrm{M}$ of each primer, $0.25 \mathrm{U}$ of red hot Taq DNA polymerase (AB Gene) and 50-100 ng of DNA template. PCR conditions were: initial denaturation at $94^{\circ} \mathrm{C}$ for $3 \mathrm{~min}, 35$ cycles of denaturation at $93^{\circ} \mathrm{C}$ for $30 \mathrm{~s}$, primer annealing for $30 \mathrm{~s}$ and primer extension at $72^{\circ} \mathrm{C}$ for $30 \mathrm{~s}$, followed by a final primer extension step at $72{ }^{\circ} \mathrm{C}$ for $7 \mathrm{~min}$. PCR products were electrophoresed on $5 \%$ denaturing polyacrylamide gels in $0.6 \times$ TBE buffer, in an automated DNA fragment analyser (Corbett Research Gel Scan V7.2.6, Real-Time Gel System) at $1200 \mathrm{~V}$ and $40^{\circ} \mathrm{C}$ for $90 \mathrm{~min}$. The sizes of PCR products were estimated with a commercial size standard (ABI PRISM, Tamra 350). Gels were visualized and alleles scored with ONED-scan V2.03 software (Scanalytics, VA, USA).

\section{Statistical analysis}

$\chi^{2}$ tests were used to discover whether or not the observed genotype ratios were statistically different to the expected genotype ratios.

\section{Results}

Inheritance of alleles in $F_{1}$ offspring

In all, 14 of the 24 ratios of genotypes in the $F_{1}$ offspring did not differ significantly from the ratios expected if inheritance was Mendelian (Table 1). Nine of these 14 ratios of genotypes were from parents that were homozygous at that locus: this resulted in offspring with identical homozygous genotypes, or with the heterozygous genotype expected when both parents are homozygous for different alleles (genotype ratio ID \#s $1,4,6,10,12,19,20,22,23$ of Table 1). The other five ratios of genotypes were from crosses where the femaleparent was heterozygous and the male parent was homozygous at that locus (genotype ratio ID \#s 2, 3, 13, 16, 24 of Table 1).
The remaining 10 ratios of genotypes differed significantly from the ratios expected when inheritance was Mendelian (genotype ratio ID \#s 5, 7, 8, 9, 11, 14, 15, 17, 18, 21 of Table 1); this was the case at all three loci. In all 10 of the ratios of genotypes where inheritance was non-Mendelian, the male-parent was heterozygous and only passed one of his two alleles to his offspring. For example, in family 3, at locus $M L 9$, the male-parent (165/168) and female-parent (169/171) had offspring with only two $(168 / 169,168 / 171)$ of the four genotypes expected if inheritance was Mendelian $\left(P=2.5 \times 10^{-05}\right)$ (genotype ratio ID \# 8, Table 1) that is, the genotypes $165 / 171$ and 165/169 were not present in the offspring of these lice.

\section{Inheritance of alleles in $\mathrm{F}_{2}$ offspring}

Both $F_{2}$ families had heterozygous male-parents from family 7 and heterozygous or homozygous femaleparents from family 8 . Inheritance was Mendelian at the two informative loci (ML9 and ML10) of family 9: (i) at locus $M L 9$, the male-parent (165/168) and femaleparent (165/165) had offspring with two genotypes (165/ 165 and 165/168), and the ratios of genotypes were as expected if inheritance was Mendelian $(P=1.000)$ (genotype ratio ID \# 26, Table 2; Supplementary Figure 1); (ii) at locus ML10, the male-parent (161/170) and femaleparent (170/170) had offspring with two genotypes (161/ 170 and 170/170), and the ratios of genotypes in the offspring were as expected if inheritance was Mendelian $(P=1.000)$ (genotype ratio ID \# 27, Table 2$)$. However, inheritance was non-Mendelian at the two informative loci (ML9 and ML10) of family 10: (i) at locus ML9, the male-parent (165/168) and female-parent (165/165) had offspring with only one genotype (165/168) (genotype ratio ID \# 29, Table 2; Supplementary Figure 2). The ratios of genotypes in these offspring differed significantly from the ratios of genotypes expected if inheritance was Mendelian $\left(P=2.21 \times 10^{-05}\right)$. Indeed, the genotype $165 / 165$ was not present in any of the offspring; (ii) at locus ML10, the male-parent (161/170) and female-parent (161/170) had offspring with only two (161/170 and 170/ $170)$ of the three genotypes expected if inheritance was Mendelian $\left(P=4.21 \times 10^{-02}\right)$ (genotype ratio ID \# 30, Table 2) that is, genotype 161/161 was not found in any of the 18 offspring.

\section{Discussion}

Our results are preliminary evidence for TRD of paternal alleles in the body louse, P. humanus. In all genotype ratios, both alleles from heterozygous female-parents were always passed to offspring. This indicates random segregation always occurred in female-parents. In contrast, alleles from heterozygous males were inherited in two ways: (i) in a Mendelian fashion, and (ii) in a nonMendelian fashion. In the case of the latter, only one of the male's two alleles was passed to his offspring.

Non-Mendelian inheritance of microsatellite alleles is often attributed to one of the two artefacts of PCR commonly associated with these genetic markers: null alleles and allelic dropout. Null alleles are alleles that are not amplified by PCR because nucleotide substitutions, insertions or deletions have occurred in one or both of the primer-annealing sites (Callen et al, 1993). If null 
Table 1 Genotypes of parents and their offspring at three microsatellite loci in eight $\mathrm{F}_{1}$ families of Pediculus humanus

\begin{tabular}{|c|c|c|c|c|c|c|c|c|}
\hline \multirow[t]{2}{*}{ Family ID \# } & \multirow[t]{2}{*}{ Locus } & \multicolumn{2}{|c|}{ Parental genotype } & \multicolumn{3}{|c|}{$F_{1}$ offspring genotype } & \multirow[t]{2}{*}{$\mathrm{P}$} & \multirow[t]{2}{*}{ Genotype ratio ID } \\
\hline & & Male & Female & $F_{1}$ & Observed number & Expected number & & \\
\hline \multirow{5}{*}{$\begin{array}{l}1 \\
(n=26)\end{array}$} & ML8 & $149 / 149$ & $149 / 149$ & $149 / 149$ & 26 & 26 & 1.000 & 1 \\
\hline & ML9 & $165 / 165$ & $167 / 169$ & $165 / 169$ & 12 & 13 & 0.695 & 2 \\
\hline & & & & $165 / 167$ & 14 & 13 & & \\
\hline & ML10 & $170 / 170$ & $170 / 175$ & $170 / 170$ & 12 & 13 & 0.695 & 3 \\
\hline & & & & $170 / 175$ & 14 & 13 & & \\
\hline \multirow{4}{*}{$\begin{array}{l}2 \\
(n=32)\end{array}$} & ML8 & $149 / 149$ & $149 / 149$ & $149 / 149$ & 32 & 32 & 1.000 & 4 \\
\hline & ML9 & $165 / 169$ & $167 / 167$ & $165 / 167$ & 0 & 16 & $1.54 \times 10^{-08}$ & 5 \\
\hline & & & & $167 / 169$ & 32 & 16 & & \\
\hline & ML10 & $170 / 170$ & $175 / 175$ & $170 / 175$ & 32 & 32 & 1.000 & 6 \\
\hline \multirow[t]{2}{*}{3} & ML8 & $131 / 149$ & $149 / 149$ & $131 / 149$ & 0 & 12 & $9.63 \times 10^{-07}$ & 7 \\
\hline & & & & $149 / 149$ & 24 & 12 & & \\
\hline \multirow[t]{8}{*}{$(n=24)$} & ML9 & $165 / 168$ & $169 / 171$ & $165 / 169$ & 0 & 6 & $2.5 \times 10^{-05}$ & 8 \\
\hline & & & & $168 / 169$ & 12 & 6 & & \\
\hline & & & & 165/171 & 0 & 6 & & \\
\hline & & & & $168 / 171$ & 12 & 6 & & \\
\hline & ML10 & $170 / 175$ & $161 / 170$ & $161 / 170$ & 12 & 6 & $2.5 \times 10^{-05}$ & 9 \\
\hline & & & & $170 / 170$ & 12 & 6 & & \\
\hline & & & & $161 / 175$ & 0 & 6 & & \\
\hline & & & & $170 / 175$ & 0 & 6 & & \\
\hline 4 & ML8 & $149 / 149$ & $149 / 149$ & $149 / 149$ & 34 & 34 & 1.000 & 10 \\
\hline \multirow[t]{4}{*}{$(n=34)$} & ML9 & $165 / 169$ & $165 / 169$ & $165 / 165$ & 0 & 8.5 & $1.3 \times 10^{-03}$ & 11 \\
\hline & & & & $165 / 169$ & 26 & 17 & & \\
\hline & & & & $169 / 169$ & 8 & 8.5 & & \\
\hline & ML10 & 170/170 & $170 / 170$ & $170 / 170$ & 34 & 34 & 1.000 & 12 \\
\hline \multirow[t]{2}{*}{5} & ML8 & $149 / 149$ & $131 / 149$ & $131 / 149$ & 19 & 15 & 0.144 & 13 \\
\hline & & & & $149 / 149$ & 11 & 15 & & \\
\hline \multirow[t]{4}{*}{$(n=30)$} & ML9 & $165 / 169$ & $165 / 165$ & $165 / 165$ & 30 & 15 & $4.32 \times 10^{-08}$ & 14 \\
\hline & & & & $165 / 169$ & 0 & 15 & & \\
\hline & ML10 & $170 / 175$ & $170 / 170$ & $170 / 170$ & 30 & 15 & $4.32 \times 10^{-08}$ & 15 \\
\hline & & & & $170 / 175$ & 0 & 15 & & \\
\hline \multirow[t]{2}{*}{6} & ML8 & $149 / 149$ & $131 / 149$ & $131 / 149$ & 8 & 7.5 & 0.796 & 16 \\
\hline & & & & $149 / 149$ & 7 & 7.5 & & \\
\hline \multirow[t]{8}{*}{$(n=15)$} & ML9 & $165 / 169$ & $165 / 167$ & $165 / 165$ & 0 & 3.75 & $1.71 \times 10^{-03}$ & 17 \\
\hline & & & & $165 / 167$ & 0 & 3.75 & & \\
\hline & & & & $165 / 169$ & 8 & 3.75 & & \\
\hline & & & & $167 / 169$ & 7 & 3.75 & & \\
\hline & ML10 & $170 / 175$ & $161 / 170$ & $161 / 170$ & 0 & 3.75 & $3.75 \times 10^{-04}$ & 18 \\
\hline & & & & $161 / 175$ & 5 & 3.75 & & \\
\hline & & & & $170 / 170$ & 0 & 3.75 & & \\
\hline & & & & $170 / 175$ & 10 & 3.75 & & \\
\hline 7 & ML8 & $149 / 149$ & $149 / 149$ & $149 / 149$ & 25 & 25 & 1.000 & 19 \\
\hline \multirow[t]{5}{*}{$(n=25)$} & ML9 & $165 / 165$ & $168 / 168$ & $165 / 168$ & 25 & 25 & 1.000 & 20 \\
\hline & ML10 & $161 / 175$ & $161 / 170$ & $161 / 161$ & 9 & 6.25 & $2.33 \times 10^{-06}$ & 21 \\
\hline & & & & $161 / 170$ & 16 & 6.25 & & \\
\hline & & & & $161 / 175$ & 0 & 6.25 & & \\
\hline & & & & $170 / 175$ & 0 & 6.25 & & \\
\hline 8 & ML8 & $149 / 149$ & $149 / 149$ & $149 / 149$ & 10 & 10 & 1.000 & 22 \\
\hline \multirow{3}{*}{$(n=10)$} & ML9 & $165 / 165$ & $165 / 165$ & $165 / 165$ & 10 & 10 & 1.000 & 23 \\
\hline & ML10 & $170 / 170$ & $161 / 170$ & $161 / 170$ & 7 & 5 & 0.206 & 24 \\
\hline & & & & $170 / 170$ & 3 & 5 & & \\
\hline
\end{tabular}

$n$, number of offspring in each family.

$P, \chi^{2}$ test probability associated with deviations between observed and expected numbers of genotypes.

Significant deviations from Mendelian expectations $(P<0.05)$ are in bold font.

alleles are present, but not accounted for, then a heterozygote with a null allele will appear to be homozygous. Null alleles are usually assigned at a microsatellite locus when the presence of a null allele in one or both parents can explain a non-Mendelian genotypic ratio observed in their offspring (Liewlaksaneeyanawin et al, 2002; de Meeus et al, 2004). Using this criterion, no null alleles were detected in any of the eight $\mathrm{F}_{1}$ and two $\mathrm{F}_{2}$ families of $P$. humanus. Mutations of primer-annealing sites between parental and offspring 
Table 2 Genotypes of parents and their offspring at three microsatellite loci in two $\mathrm{F}_{2}$ families of Pediculus humanus

\begin{tabular}{|c|c|c|c|c|c|c|c|c|}
\hline \multirow[t]{2}{*}{ Family ID \# } & \multirow[t]{2}{*}{ Locus } & \multicolumn{2}{|c|}{ Parental genotype } & \multicolumn{3}{|c|}{$F_{2}$ offspring genotype } & \multirow[t]{2}{*}{$\mathrm{P}$} & \multirow[t]{2}{*}{ Genotype ratio ID \# } \\
\hline & & Male & Female & $F_{2}$ & Observed number & Expected number & & \\
\hline 9 & ML8 & $149 / 149$ & $149 / 149$ & $149 / 149$ & 30 & 30 & 1.000 & 25 \\
\hline \multirow[t]{4}{*}{$(n=30)$} & ML9 & $165 / 168$ & $165 / 165$ & $165 / 165$ & 15 & 15 & 1.000 & 26 \\
\hline & & & & $165 / 168$ & 15 & 15 & & \\
\hline & ML10 & $161 / 170$ & $170 / 170$ & $161 / 170$ & 15 & 15 & 1.000 & 27 \\
\hline & & & & $170 / 170$ & 15 & 15 & & \\
\hline 10 & ML8 & $149 / 149$ & $149 / 149$ & $149 / 149$ & 18 & 18 & 1.000 & 28 \\
\hline \multirow[t]{5}{*}{$(n=18)$} & ML9 & $165 / 168$ & $165 / 165$ & $165 / 165$ & 0 & 9 & $2.21 \times 10^{-05}$ & 29 \\
\hline & & & & $165 / 168$ & 18 & 9 & & \\
\hline & ML10 & $161 / 170$ & $161 / 170$ & $161 / 161$ & 0 & 4.5 & $4.21 \times 10^{-02}$ & 30 \\
\hline & & & & $161 / 170$ & 11 & 9 & & \\
\hline & & & & $170 / 170$ & 7 & 4.5 & & \\
\hline
\end{tabular}

$n$, number of offspring in each family.

$P, \chi^{2}$ test probability associated with deviations between observed and expected numbers of genotypes.

Significant deviations from Mendelian expectations $(P<0.05)$ are in bold font.

generations, however, could lead to null alleles in their offspring, and the apparent loss of alleles transmitted from parents to offspring. However, this cannot account for the non-Mendelian inheritance that we found at loci in families where offspring were all heterozygous (genotype ratio ID \#s 5, 8, 17, 18 of Table 1 and genotype ratio ID \# 29 of Table 2). The presence of null alleles at the three microsatellite loci we studied in P. humanus is, therefore, unlikely.

Allelic dropout is the preferential amplification of only one of two alleles at a locus. Allelic dropout leads to scoring individuals as homozygotes when they are heterozygotes (Miller et al, 2002). Allelic dropout is most likely when DNA of low quality, for example DNA from museum specimens and noninvasive sources, is used in PCR (Gagneux et al, 1997). Like null alleles, however, allelic dropout cannot account for the non-Mendelian inheritance observed in families of $P$. humanus where TRD of paternal alleles occurred and all offspring had heterozygous genotypes (genotype ratio ID \#s 5, 8, 17, 18 of Table 1 and genotype ratio ID \# 29 of Table 2). In addition, repeated amplification of microsatellite loci from individual lice at a range of template DNA concentrations gave consistent results.

Given the lack of evidence for null alleles and allelic dropout, the pattern of inheritance we discovered is evidence of extreme TRD of paternal alleles. Unlike most reports of TRD, a single allele was inherited exclusively in P. humanus when fathers were heterozygous. Elimination of complete sets of chromosomes from males has been observed in several insect species. For example, some males of the parasitic wasps Nasonia vitripennis and Trichogramma kaykai have supernumerary B (PSR paternal sex ratio) chromosomes that cause the loss of all the paternal chromosomes except themselves in sperm transmitted from haploid males (Werren and Stouthamer, 2003). In another example, half of the accessory chromosomes are eliminated from the germ line during sex determination the sciarid fly, Sciaria cophrilia (Rieffel and Crouse, 1966). The pattern of inheritance we discovered in $P$. humanus, however, differs from these examples in that: (i) there were no statistically significant alterations in the sex ratios of families where TRD occurred (data not shown); and (ii) male $P$. humanus are diploid as evidenced by the transmission of two alleles.

The pattern of inheritance in P. humanus families where TRD of paternal alleles occurred is similar to that described for a number of insect and mite species with paternal genome elimination (PGE) (Perrot-Minnot $e t$ al, 2000; Normark, 2003). In females of species with PGE, oogenesis is normal, with random segregation and assortment of chromosomes. Males, however, transmit to their offspring only their maternal set of chromosomes, because their paternal set are eliminated at some stage during development. In most cases, the paternal chromosomes are eliminated by meiotic drive following nonindependent assortment of maternal and paternal chromosomes during spermatogenesis (Bongiorni et al, 2004). In family 10 of our study, the $F_{1}$ male did not pass to his $F_{2}$ offspring alleles of grand-paternal origin at the two informative loci: $M L 9$ ( $165 \mathrm{bp}$ allele) and ML10 (161 bp allele) that is, the alleles the male received from his $P_{1}$ father (see genotype ratio ID \#s 20, 21 of Table 1 and genotype ratio ID \#s 29, 30 of Table 2). This suggests a way of distinguishing paternally derived from maternally derived chromosomes in P. humanus, and a way of eliminating paternally derived chromosomes prior to fertilisation of the $\mathrm{F}_{2}$. Changes in chromatin structure (ie heterochromatisation) and imprinting, as in lecanoid scale insects (Bongiorni and Prantera, 2003), are possible mechanisms for this process in P. humanus.

The pattern of inheritance we discovered in P. humanus is unlike other types of PGE because we did observe one heterozygous male in which alleles segregated in a Mendelian fashion ( $F_{1}$ male - Family 9, Table 2). Inheritance in P. humanus males is therefore similar to TRD such as segregation distorter in D. melanogaster (Lyttle, 1993), and the t-locus in Mus domesticus and M. musculus (Ardlie and Silver, 1996) in that one allele is transmitted predominantly (95-99\%) to offspring in some males. In this study, the only P. humanus heterozygous male in which alleles segregated in a Mendelian fashion ( $F_{1}$ male of family 9$)$ was from a family in which TRD of paternal alleles occurred (family 7). In contrast, in the other heterozygous male from this family $\left(F_{1}\right.$ male 
of family 10), paternal alleles were eliminated. This suggests that the female-parent of these two males may have been heterozygous for a genetic element that eliminates alleles in male $P$. humanus. Intriguingly, maternally inherited genetic elements causing elimination of paternal chromosomes in males have been hypothesised as a mechanism that could lead to the evolution of PGE (Brown, 1964). Another hypothesis that could explain the elimination of alleles in some male $P$. humanus but not others, is the presence of a B chromosome that eliminates paternally derived chromosomes; but with imperfect transmission efficiency so that some males avoid the B chromosome but others do not. Males with B chromosomes that were transmitted with their maternal chromosomes have been observed in the mealybug Pseudococcus affinis, an insect which has PGE (Nur and Brett, 1988). Although B chromosomes have not been observed in $P$. humanus, they have been observed in other lice including the pig louse, Haematopinus suis (Tombesi and Papeschi, 1993). Further genetic characterisation of body lice by additional crosses should help to discern whether or not such a genetic element is segregating in the Orlando strain of P. humanus.

Significantly, spermatogenesis in P. humanus is very unusual and provides a setting for elimination of chromosomes or alleles (Hindle and Pontecorvo, 1942; White, 1973). In P. humanus the somatic (2n) number is 12 in both sexes. In $P$. humanus males, the four primary spermatids from meiosis of one primary spermatocyte, divide mitotically four times to give a cyst of 64 secondary spermatids. The secondary spermatids then go through an unusual fifth division that is unequal with respect to the cytoplasm (Doncaster and Cannon, 1919). In each secondary spermatid a small-nucleated 'bud' containing six chromosomes is cutoff from the main cell. Only the main cell forms sperm, the bud (pycnotic nuclei) degenerates (Hindle and Pontecorvo, 1942). This results in a cyst of 64 tertiary spermatids that develop into active sperm, and 64 pycnotic nuclei that degenerate. It is possible that this fifth division may be nonrandom in $P$. humanus, resulting in specific chromosomes of paternal or allelic-specific origin being eliminated. Only certain chromosomes would, therefore, be present in viable sperm. We hypothesise that the genetic mechanism that acts to eliminate or retain chromosomes in P. humanus occurs during this stage of spermatogenesis.

TRD in P. humanus is unlikely to be due to differential survival of diploid zygotes with alternative alleles, because $90 \%$ of all the eggs laid in each family hatched (data not shown). Therefore, TRD of paternal alleles in $P$. humanus most probably occurs by meiotic drive of chromosomes during spermatogenesis, or postmeiotic selection prior to fertilisation. Since the three microsatellite loci (ML8, ML9 and ML10) we used have not been mapped to chromosomes of P. humanus, it is not possible to tell if this system of TRD involves a single chromosome or an entire parental set of chromosomes. Future studies should therefore aim to map these loci and other genetic markers (eg SNPs and RAPDs), to create a linkage map for $P$. humanus. This method has been used successfully to characterise novel meiotic drive loci in other species (Fishman and Willis, 2005). Coupled with cytogenetics, this approach may shed light on the mechanism of TRD in this insect.

\section{Acknowledgements}

We thank Dr Natalie Leo, Ms Maryam Ashrafi, Ms Jing $\mathrm{Ma}$ and Professor Jane Hughes for enthusiastic technical assistance and expert advice.

\section{References}

Ardlie K, Silver LM (1996). Recent evolution of mouse $t$ haplotypes at polymorphic microsatellites associated with the $t$ complex responder (Tcr) locus. Genet Res 67: 1-10.

Bongiorni S, Prantera G (2003). Imprinted facultative heterochromatization in mealybugs. Genetica 117: 271-279.

Bongiorni S, Fiorenzo P, Pippoletti D, Prantera G (2004). Inverted meiosis and meiotic drive in mealybugs. Chromosoma 112: 331-341.

Brown SW (1964). Automatic frequency response in the evolution of male haploidy and other coccid chromosome systems. Genetics 49: 797-817.

Callen DF, Thompson AD, Shen Y, Phillips HA, Richards RI, Mulley JC et al (1993). Incidence and origin of 'null' alleles in the (AC)n microsatellite markers. Am J Hum Genet 52: 922-927.

Culpepper GH (1944). The rearing and maintenance of a laboratory colony of the body louse. Am J Trop Med 24: 327-329.

Culpepper GH (1946). Factors influencing the rearing and maintenance of a laboratory colony of the body louse. J Econ Entomol 39: 472-474.

Culpepper GH (1948). Rearing and maintaining a laboratory colony of body lice on rabbits. Am J Trop Med 28: 499-504.

de Meeus T, Humair PF, Grunau C, Delaye C, Renaud F (2004). Non-Mendelian transmission of alleles at microsatellite loci: an example in Ixodes ricinus, the vector of Lyme disease. Int J Parasitol 34: 943-950.

Doncaster L, Cannon HG (1919). On the spermatogenesis of the louse (Pediculus corporis and P. capitis), with some observations on the maturation of the egg. Quart J Micr Sci 64: 303-328.

Fishman L, Kelly AJ, Morgan E, Willis JH (2001). A genetic map in the Mimulus guttatus species complex reveals transmission ratio distortion due to heterospecific interactions. Genetics 159: 1701-1716.

Fishman L, Willis JH (2005). A novel meiotic drive locus almost completely distorts segregation in Mimulus (monkeyflower) hybrids. Genetics 169: 347-353.

Gagneux P, Boesch C, Woodruff DS (1997). Microsatellite scoring errors associated with noninvasive genotyping based on nuclear DNA amplified from shed hair. Mol Ecol 6: 861-868.

Hindle E, Pontecorvo G (1942). Mitotic divisions following meiosis in Pediculus corporis males. Nature 149: 668.

Leo NP, Hughes JM, Yang X, Poudel SKS, Brogden WG, Barker SC (2005). The head and body lice of humans are genetically distinct (Insecta: Phthiraptera, Pediculidae): evidence from double infestations. Heredity 95: 34-40.

Liewlaksaneeyanawin C, Ritland CE, El-Kassaby YA (2002). Inheritance of null alleles for microsatellites in the white pine weevil (Pissodes strobi [Peck] [Coleoptera: Curculionidae]). J Hered 93: 67-70.

Lyttle TW (1991). Segregation distorters. Annu Rev Genet 25: 511-557.

Lyttle TW (1993). Cheaters sometimes prosper: distortion of Mendelian segregation by meiotic drive. Trends Genet 9: 205-210.

Miller CR, Joyce P, Waits LP (2002). Assessing allelic dropout and genotype reliability using maximum likelihood. Genetics 160: $357-366$

Normark BB (2003). The evolution of alternative genetic systems in insects. Annu Rev Entomol 48: 397-423.

Nur U, Brett BL (1988). Genotypes affecting the condensation and transmission of heterochromatic B chromosomes in the mealybug Pseudococcus affinis. Chromosoma 96: 205-212.

Pardo-Manuel de Villena F, de la Casa-Esperón E, Briscoe TL, Sapienza C (2000). A genetic test to determine the origin of the maternal 1: meiotic drive at the mouse $\mathrm{Om}$ locus. Genetics 154: 333-342. 
Perrot-Minnot MJ, Lagnel J, Migeon A, Navajas M (2000). Tracking paternal genes with DALP markers in a pseudoarrhenotokous reproductive system: biparental transmission but haplodiploid-like inheritance in the mite Neoseiulus californicus. Heredity 84: 702-709.

Rieffel SM, Crouse HV (1966). The elimination and differentiation of chromosomes in the germ line of Sciaria. Chromosoma 19: $231-276$.
Tombesi ML, Papeschi AG (1993). Meiosis in Haematopinus suis and Menacanthus stramineus (Phthiraptera, Insecta). Hereditas 119: $31-38$.

Werren JH, Stouthamer R (2003). PSR (paternal sex ratio) chromosomes: the ultimate selfish genetic elements. Genetica 117: 85-101.

White MJD (1973). Animal Cytology and Evolution. 3rd edn. Cambridge University Press: UK.

Supplementary Information accompanies the paper on the Heredity website (http://www.nature.com/hdy) 